\title{
Controlled Assembly of Hybrid Bulk-Heterojunction Solar Cells by Sequential
}

\section{Deposition}

\author{
Ilan Gur ${ }^{1,3}$, Neil Fromer ${ }^{1,3}$, and A. Paul Alivisatos ${ }^{*}, \mathbf{1 , 2}$ \\ ${ }^{\mathbf{1}}$ Materials Science Division, Lawrence Berkeley National Laboratory, Berkeley CA \\ 94720 \\ ${ }^{2}$ Department of Chemistry, University of California, Berkeley CA 94720 \\ ${ }^{3}$ Department of Materials Science and Engineering, University of California, Berkeley \\ CA 94720 \\ * To whom correspondence should be addressed. Email: alivis@berkeley.edu
}




\begin{abstract}
This work presents a technique to create ordered and easily characterized hybrid nanocrystal-polymer composites by sequential deposition of tetrapod-shaped cadmium telluride (CdTe) nanocrystals and poly(3-hexlythiophene). With controlled fabrication and composite morphology, these devices offer several advantages over traditional codeposited hybrid cells, and provide a model system for detailed investigation into the operation of bulk-heterojunction cells.
\end{abstract}


Excitonic solar cells hold great promise for enabling the production of large-scale solar power at low costs ${ }^{1}$. The most efficient of these cells utilize a type II donor-acceptor heterojunction to facilitate the separation of otherwise bound excitons ${ }^{2}$. Donor-acceptor cells based on simple planar architectures are limited in thickness, and in turn efficiency, by small exciton diffusion lengths. Yet when donor and acceptor materials are blended to create an interspersed or bulk heterojunction, cells can be made thick enough for strong absorption while still allowing for efficient charge separation ${ }^{3,4}$.

Nanocomposite bulk-heterojunctions constitute the most efficient class of polymer-based solar cells; however, their superior performance is contingent upon on a complex architecture that is extremely difficult to control ${ }^{5-10}$. The ideal nanocomposite solar cell architecture allows the small domain sizes and large interfacial area required for optimal charge separation while still providing directed pathways for rapid carrier conduction to a set of carrier-selective electrodes. The majority of actual bulk heterojunction cells are fabricated by co-deposition of a random donor/acceptor composite from solution, and though simple and inexpensive, this process offers little control over parameters such as blend morphology and electrode contacts, which are critical in determining the ultimate cell performance. Even characterizing the structure of co-deposited films can prove a difficult task, requiring one to rely on indirect indicators such as surface roughness or final device performance to infer the quality of the blend ${ }^{11}$.

The limitations of co-deposited bulk heterojunction blends are readily evident in traditional hybrid polymer-nanocrystal solar cells, which consist of a hole-conducting polymer, poly(3-hexylthiophene) (P3HT), blended with inorganic semiconductor 
nanocrystals ${ }^{12,13}$. Typically, hybrid composites are co-deposited via spin-casting from a solution containing both pyridine and chloroform. An optimal mixture of these two solvents can stabilize both the organic and inorganic phases, thereby allowing for deposition of an intimate random blend film. Unfortunately, the perfect solvent mixture is hard to ascertain, and the ultimate blend morphology remains highly sensitive to solvent concentrations as well as variations in the nanocrystal synthesis and processing 11,14. Blend defects such as islands and aggregation are common, and while varying the type and proportions of solvents used can help mitigate their effects and improve device performance $^{15}$, the random nature of the blend film inhibits alignment of the blend domains, interfering with the conduction and extraction of photoexcited carriers (Fig 1A). Here, we present a technique to create ordered and easily characterized hybrid composites by sequential deposition of tetrapod-shaped cadmium telluride (CdTe) nanocrystals and P3HT.

We have previously demonstrated that tetrapod-shaped CdTe nanocrystals can be readily synthesized and prepared in solution, with a wide range of control over arm diameter and length ${ }^{16}$. We now show that such nanocrystals can be used as a template for creating ordered composite structures with controlled dispersion. Figure 1 schematically illustrates the desired architecture (1B), and presents a transmission electron micrograph of the tetrapod nanocrystals utilized in this study (1C). The composite fabrication approach is simple. First, nancrystal tetrapods are deposited on an electrode surface. Deposition can be achieved via spin-casting or, alternatively, an amphiphilic linker molecule may be used to anchor the nanocrystals to the desired surface ${ }^{17}$. At this stage, the nanocrystal density and dispersion on the surface can be easily characterized by 
scanning electron microscopy. The unique tetragonal structure of the nanocrystals gives rise to a natural ordering in the deposited films; three arms of each tetrapod contact on the substrate at its base while the fourth arm points up, perpendicular to the substrate. This ordering remains evident in the composite films, created by spin-casting a polymer from its optimal solvent to cover the nanocrystal film.

Fig 2 presents SEM micrographs illustrating the wide range of control over nanocrystal dispersions achievable by various deposition methods. Imaging the array of nanocrystals created by any one deposition approach allows for a direct measure of the crystal loading and dispersion that will appear in the ultimate blend film. In Fig 2A, CdTe tetrapods are anchored to a gold substrate via the linker molecule hexane dithiol. Figs 2B-D show similar arrays of nanocrystals, here anchored to ITO substrates by means of carboxylic acid or siloxane functionalities. After depositing one layer of nanocrystals, it is possible to anchor a second layer to the surface (Fig 2E). Repeating this procedure can yield even denser nanocrystals arrays. In choice of linker molecule and number of deposition steps, one can thus tailor the loading of nanocrystals that will appear in the final composite film. Finally, extremely dense nanocrystals films can be deposited via spin-casting (Fig 2F).

Figure 3 shows several hybrid composites created by this approach. Since a single template of nanocrystals deposited on ITO (Fig 3A) was used for all composite films, the loading and dispersion of nanocrystals remain constant across the series. However, the figure illustrates a further degree of control afforded in creating nanocrystal-polymer composites by this approach. By varying the concentration of the solution from which the polymer is spin-cast, the height to which the polymer matrix covers the original 
nanocrystal template array can be easily adjusted. Composites can thus be created in which much of the nanocrystals remain exposed (Fig 3B), or only the tips of the nanocrystals remain exposed (Fig 3C). Increasing the concentration of P3HT yields films in which the nanocrystals are embedded within the polymer matrix (Fig 3D). Finally, the most concentrated polymer solutions yield composites with nanocrystals deeply embedded (Fig 3E). This wide range of architectures is fabricated simply by varying the concentration of spin-cast P3HT in solution from $5 \mathrm{mg} / \mathrm{ml}$ to $15 \mathrm{mg} / \mathrm{ml}$. Such control is of particular interest in the fabrication of electronic devices, allowing for intentional and determinate contact by the top electrode to either only one particular phase or to both phases simultaneously.

Preliminary studies of photovoltaic devices based on the controlled composites shown in Fig. 3 show well-defined trends as a function of polymer deposition. Device characteristics for this set of cells appear in Fig 4. In Fig 4A, photoresponse spectra are shown as a function of P3HT thickness. When the tetrapods are only partially covered, we see a large photocurrent peak corresponding to the $\mathrm{P} 3 \mathrm{HT}$ absorption, as well as a small tail resulting from the CdTe absorption. Yet upon fully covering the tetrapods, the quantum efficiency drops dramatically, as electrons are blocked from being transported to the anode. The photocurrent tail from CdTe absorption disappears entirely in these cases, and the photocurrent that does remain is likely produced at the $\mathrm{P} 3 \mathrm{HT} / \mathrm{electrode}$ interfaces. The current-voltage data in Fig 4B are consistent with this picture, showing that an increase in P3HT covering on the tetrapods results in smaller photocurrents. Finally, the data reveal a complementary trend in the open circuit voltage, which increases with increased coating of the tetrapods. Direct contact of the CdTe to both electrodes results 
in shunting, which is mitigated by a thin overlayer of P3HT. Indeed, the open circuit voltage saturates when the tetrapods are completely covered.

The nanocomposite solar cells described here offers several potential advantages over those fabricated by random co-deposition: the particles are naturally aligned toward the electrodes, loading and dispersion are easily controlled and characterized, the organic and inorganic phases are deposited from their preferred solvents rather than a suboptimal cosolution, and exposure of the nanocrystals phase to the top electrode can be controlled. At this early stage, the efficiency of these devices remains rather low, suggesting that further variations of composition, morphology, and annealing are needed. The morphology may be improved by using 3-dimensional nanoparticles with greater degrees of branching, thereby better filling space in the device while retaining a well-ordered system $^{18}$. 


\section{Acknowledgements}

This work was supported by the Director, Office of Energy Research, Office of Science, Division of Materials Sciences, of the U.S. Department of Energy under Contract No. DE-AC02-05CH11231. I.G. further acknowledges the National Science Foundation for support under a Graduate Research Fellowship, and N.F. is partially supported by a Berkeley/ITRI (Industrial Technology Research Institute, Taiwan) Research Center Fellowship. The authors wish to offer particular thanks to the following individuals for research support and valuable discussion: Delia Milliron, Kevin Sivula, Haitao Liu, and James Wang.

Supporting Information Available: Detailed information on materials and methods is available free of charge via the Internet at http://pubs.acs.org. 
(1) Shaheen, S. E.; Ginley, D. S.; Jabbour, G. E. Mrs Bulletin 2005, 30, 10.

(2) Gregg, B. A.; Hanna, M. C. Journal of Applied Physics 2003, 93, 3605.

(3) Halls, J. J. M.; Walsh, C. A.; Greenham, N. C.; Marseglia, E. A.; Friend, R. H.; Moratti, S. C.; Holmes, A. B. Nature 1995, 376, 498.

(4) Yu, G.; Gao, J.; Hummelen, J. C.; Wudl, F.; Heeger, A. J. Science 1995, $270,1789$.

(5) Ma, W. L.; Yang, C. Y.; Gong, X.; Lee, K.; Heeger, A. J. Advanced Functional Materials 2005, 15, 1617.

(6) Chirvase, D.; Parisi, J.; Hummelen, J. C.; Dyakonov, V. Nanotechnology 2004, 15, 1317.

(7) Hoppe, H.; Niggemann, M.; Winder, C.; Kraut, J.; Hiesgen, R.; Hinsch, A.; Meissner, D.; Sariciftci, N. S. Advanced Functional Materials 2004, 14, 1005.

(8) Hoppe, H.; Sariciftci, N. S. Journal of Materials Chemistry 2006, 16, 45.

(9) Watkins, P. K.; Walker, A. B.; Verschoor, G. L. B. Nano Letters 2005, 5 , 1814.

(10) Coakley, K. M.; McGehee, M. D. Applied Physics Letters 2003, 83, 3380.

(11) Huynh, W. U.; Dittmer, J. J.; Libby, W. C.; Whiting, G. L.; Alivisatos, A. P. Advanced Functional Materials 2003, 13, 73.

(12) Huynh, W. U.; Dittmer, J. J.; Alivisatos, A. P. Science 2002, 295, 2425.

(13) Milliron, D. J.; Gur, I.; Alivisatos, A. P. Mrs Bulletin 2005, 30, 41.

(14) Liu, J. S.; Tanaka, T.; Sivula, K.; Alivisatos, A. P.; Frechet, J. M. J. Journal of the American Chemical Society 2004, 126, 6550.

(15) Sun, B. Q.; Snaith, H. J.; Dhoot, A. S.; Westenhoff, S.; Greenham, N. C. Journal of Applied Physics 2005, 97.

(16) Manna, L.; Milliron, D. J.; Meisel, A.; Scher, E. C.; Alivisatos, A. P. Nature Materials 2003, 2, 382.

(17) Liu, H. T.; Alivisatos, A. P. Nano Letters 2004, 4, 2397.

(18) Kanaras, A. G.; Sonnichsen, C.; Liu, H. T.; Alivisatos, A. P. Nano Letters 2005, 5, 2164. 
Figure 1: A simple schematic juxtaposes the cross-sectional morphology of traditional hybrid nanocrystal-polymer solar cells (A) with that of ordered hybrid devices based on tetrapod nanocrystals (B). Defects such as islands and aggregates detrimental to the performance of conventional hybrid cells are eliminated in ordered arrays of tetrapods, which provide directed transport to electrodes. In $\mathbf{C}$, a transmission electron micrograph shows the tetrapod nanocrystals used in this study. Scale bar, 100nm.

Figure 2: Scanning electron micrographs show arrays of CdTe tetrapods used as templates for hybrid composites. Tetrapods may be anchored to gold substrates by hexanedithiol (A) or to ITO substrates with the following linker molecules: 3mercaptopropionic acid (B), 11-mercaptoundecanoic acid (C), mercaptopropyl trimethoxysilane (D). In (E), a second layer of tetrapods has been anchored to the film shown in (D). Finally, spincasting can be used to produce very dense template films on a variety of substrates $(\mathbf{F})$. Scale bar $500 \mathrm{~nm}$.

Figure 3: Scanning electron micrographs of an original template tetrapod array on ITO (A) and several composites produced by subsequent deposition of P3HT. P3HT was spincast from solution in chloroform with concentrations $5 \mathrm{mg} / \mathrm{ml}(\mathbf{B}), 7.5 \mathrm{mg} / \mathrm{ml}(\mathbf{C})$, $10 \mathrm{mg} / \mathrm{ml}$ (D), and $15 \mathrm{mg} / \mathrm{ml}$, allowing for controlled thickness and exposure of the inorganic nanocrystals. Scale bar $200 \mathrm{~nm}$. 
Figure 4: Normalized photoaction spectra (A) and current-voltage characteristics (B) of devices produced from ordered composites show a systematic dependence of photocurrent and open circuit voltage on controlled parameters such as P3HT thickness and electrode contact. Devices depicted in Fig 3 (B-E) are represented by $\diamond, \square, \times$. And + respectively. $100 \mathrm{~nm} \mathrm{Al} \mathrm{was} \mathrm{used} \mathrm{as} \mathrm{a} \mathrm{top} \mathrm{electrode.}$ 
Figure 1

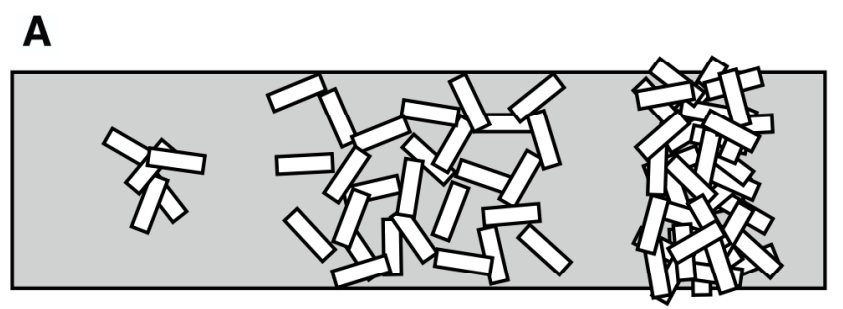

B

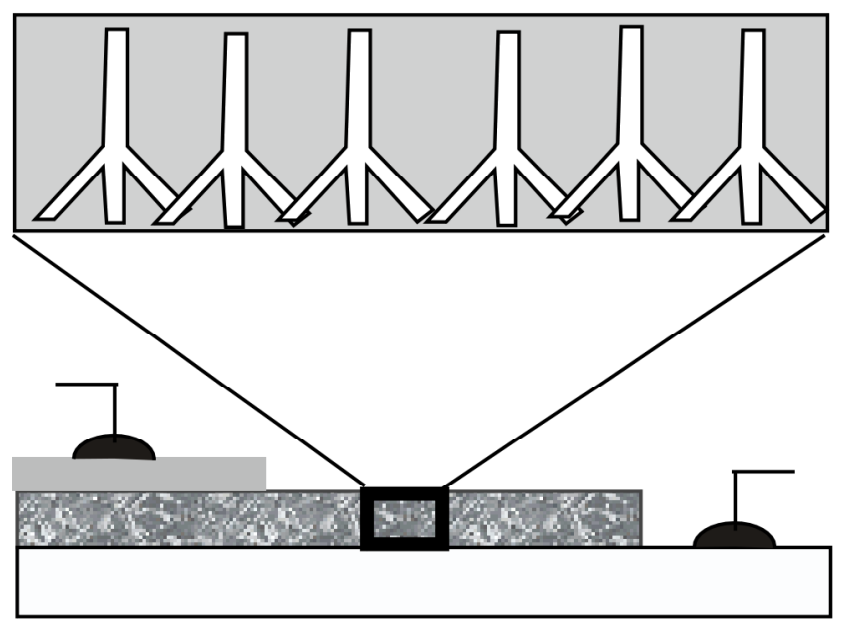

C

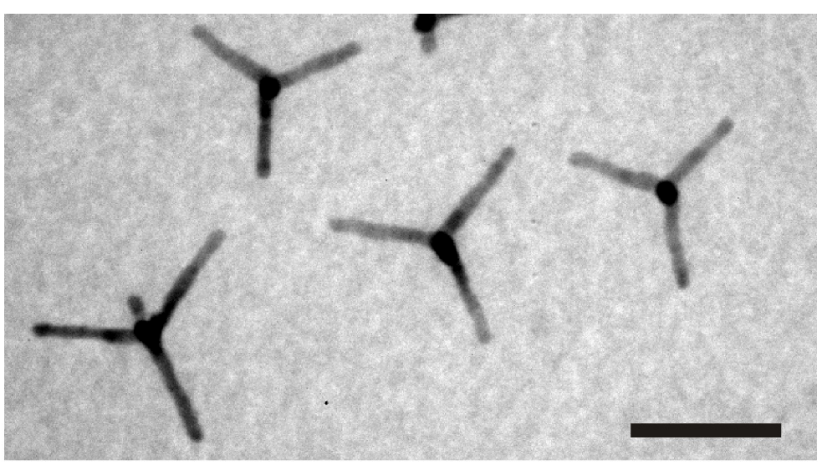


Figure 2
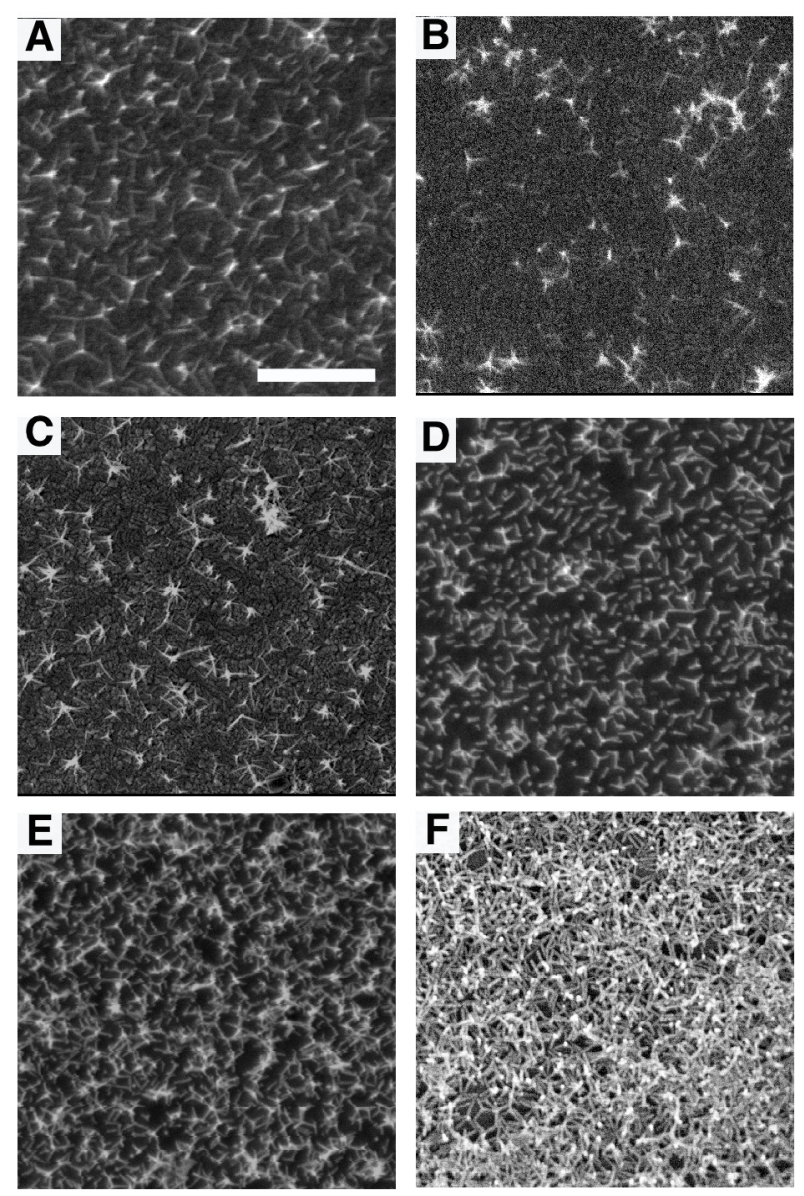
Figure 3

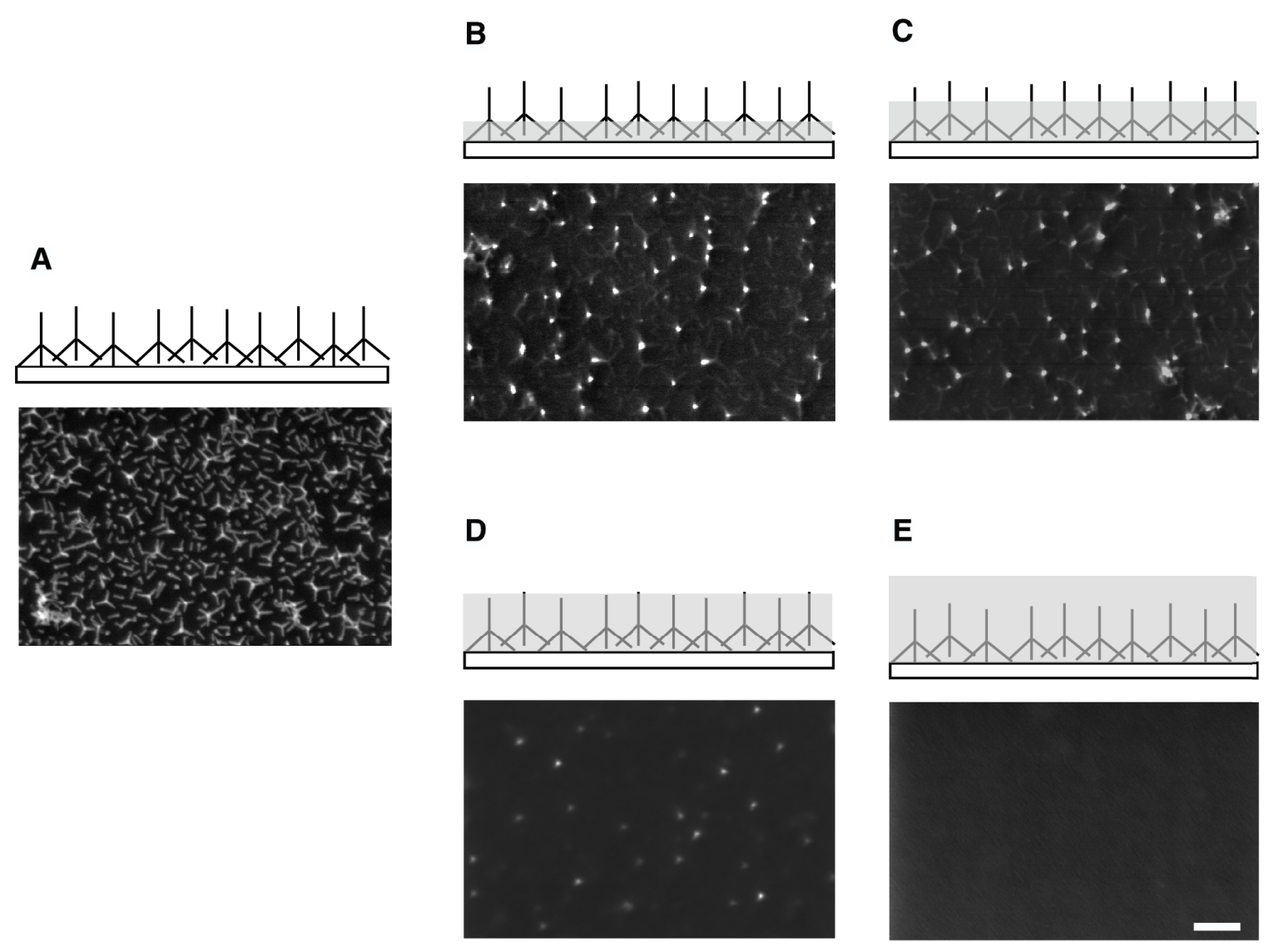


Figure 4
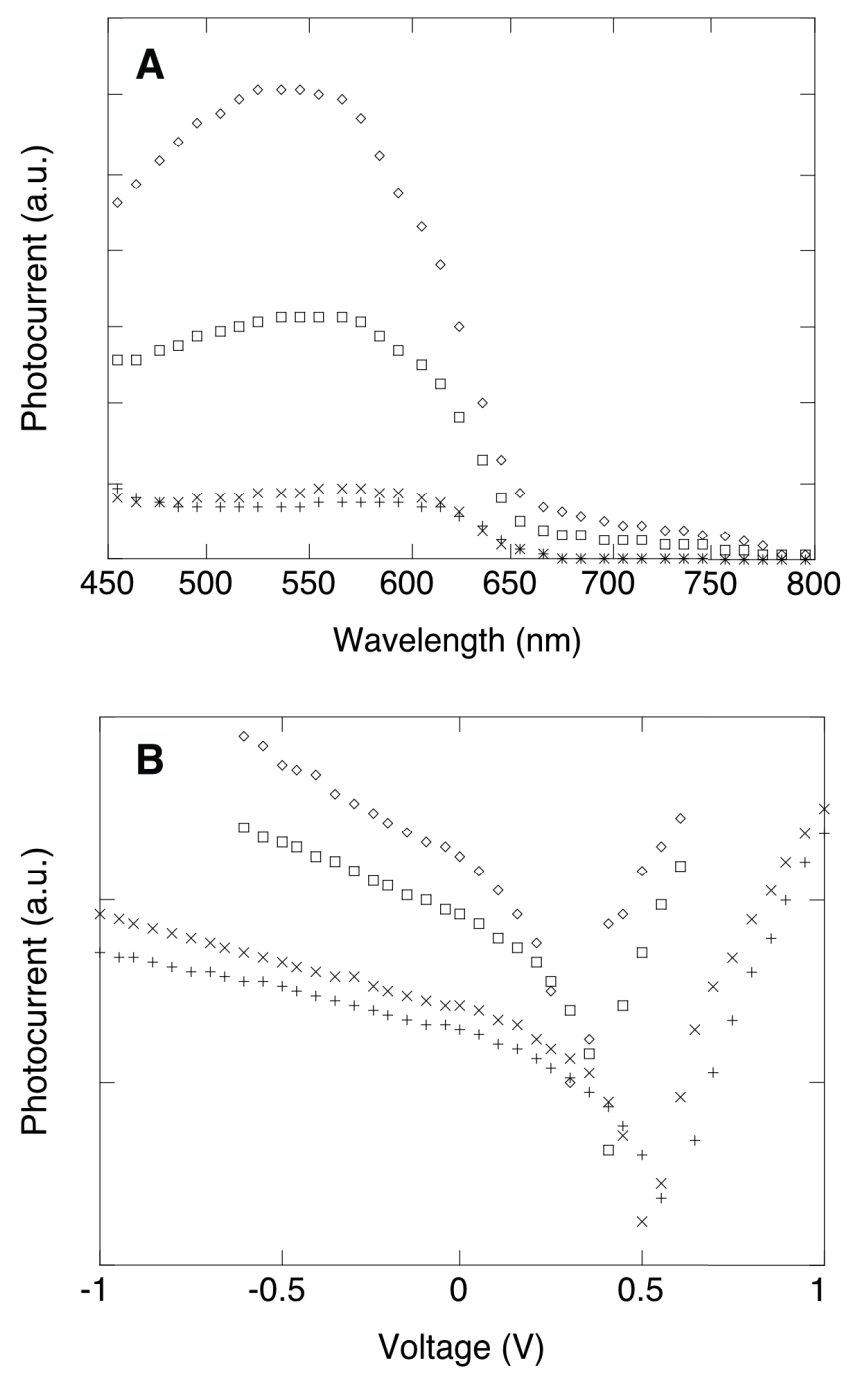
Table of Contents Image

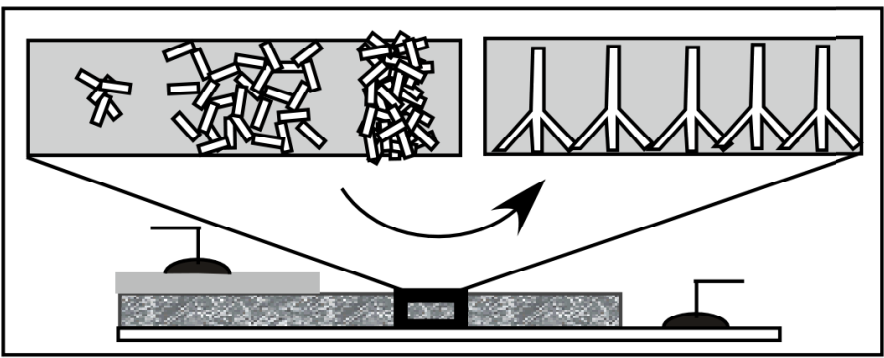

